\title{
Inexpensive Measuring System for the Characterization of Organic Transistors
}

\author{
Clara Pérez-Fuster, José Vicente Lidón-Roger, Laura Contat-Rodrigo, \\ and Eduardo García-Breijo
}

\author{
Group of Electronic Development and Printed Sensors (GEPDS), \\ Instituto Interuniversitario de Investigación de Reconocimiento Molecular y Desarrollo Tecnológico (IDM), \\ Universitat Politècnica de València, Universitat de València, Camino de Vera, s/n, 46022 Valencia, Spain
}

Correspondence should be addressed to Eduardo García-Breijo; egarciab@eln.upv.es

Received 22 August 2017; Revised 24 November 2017; Accepted 10 December 2017; Published 18 February 2018

Academic Editor: Andreas Schütze

\begin{abstract}
Copyright ( 2018 Clara Pérez-Fuster et al. This is an open access article distributed under the Creative Commons Attribution License, which permits unrestricted use, distribution, and reproduction in any medium, provided the original work is properly cited.
\end{abstract}

\begin{abstract}
A measuring module has been specifically designed for the electrical characterization of organic semiconductor devices such as organic field effect transistors (OFETs) and organic electrochemical transistors (OECTs) according to the IEEE 1620-2008 standard. This device has been tested with OFETs based on 6,13-bis(triisopropylsilylethinyl)pentacene (TIPS-pentacene). The measuring system has been constructed using a NI-PXIe-1073 chassis with integrated controller and two NI-PXI-4132 programmable high-precision source measure units (SMUs) that offer a four-quadrant $\pm 100 \mathrm{~V}$ output, with resolution down to 10 pA. LabVIEW ${ }^{\mathrm{TM}}$ has been used to develop the appropriate program. Most of the main OFET parameters included in the IEEE 1620 standard can be measured by means of this device. Although nowadays expensive devices for the characterization of Si-based transistors are available, devices for the characterization of organic transistors are not yet widespread in the market. Fabrication of a specific and flexible module that can be used to characterize this type of transistors would provide a powerful tool to researchers.
\end{abstract}

\section{Introduction}

Latest trends in the electronics industry are focused on looking for alternatives to inorganic semiconductors (based on silicon) by organic semiconductors in some applications, namely, transistors $[1,2]$, light-emitting diodes [3, 4], photovoltaic cells $[5,6]$, displays [7], sensors [8,9], and even integrated circuits $[1,10]$. These new devices use the same circuits and mechanical structures than inorganic devices, with the advantage of being easier to develop and setup by conventional chemical techniques. Standards describing the fabrication procedures of these organic devices already exist, and substrates and other raw materials for their fabrication are commercially available at low cost [11-13].

Development of these new electronic devices requires their electrical characterization by means of the appropriate equipment [14]. Devices used so far for this purpose are those traditionally used for the characterization of Si-based transistors, since the operation of organic transistors can be explained on the basis of the Si semiconductors theory, with a good approach. However, it is of great interest to develop devices that allow organic transistors to be specifically studied and that could contribute therefore to improve the understanding of their operation. Characterization of these new electronic devices is hindered by the very low electrical current they provide (around $10^{-6} \mathrm{~A}$ ), with the further difficulty that they usually require high voltages (tens of volts). Moreover, commercial systems that fulfill these characteristics are very expensive.

This work aims at describing a measuring device that has been specifically designed to study organic transistors, using exclusively low-cost commercial components and a software that has been specially developed for this application. The device described here has great flexibility, since it can be used 
for different types of transistors. Finally, this module has been used to characterize an organic field effect transistor (OFET) based on TIPS-pentacene.

OFETs have attracted increasing attention in the past decades due to their potential application as sensor systems for the detection of chemical species [15]. OFETs show superior performance compared to resistor-type, amperometric, and potentiometric sensors, and the amplification inherent to transistors enhances their sensitivity [16]. Moreover, OFETs are low cost and flexible devices that offer several opportunities for selectivity, among others, to polar vapors [17] and vapors of small-molecule analytes [18].

\section{Materials and Methods}

2.1. OFET Fabrication. Prefabricated OFET test chips based on low-density silicon oxide $\left(\mathrm{SiO}_{2}\right)$ substrate have been used (Ossila Ltd., Sheffield, UK). Each unit consists of 5 bottomcontact/bottom-gate transistors, with $30 \mu \mathrm{m}$ channel length and $1 \mathrm{~mm}$ channel width, gold/chrome electrodes in the active area and chrome/aluminum electrodes in the contact pads. The substrate consists of a highly p-doped (Boron) silicon wafer coated with $100 \mathrm{~nm}$ thick layer of $\mathrm{SiO}_{2}$ on both sides (Table 1). The doped silicon and the oxide layer provide the gate and the dielectric, respectively. A detailed schematic of the substrate is displayed in Figure 1.

TIPS-pentacene (6,13-bis (triisopropylsilylethinyl) pentacene) has been used as a semiconductor in these OFETs [19]. This compound is a dark blue solid that is commercially available in the form of small crystals that can be dissolved in anisole, decane, toluene, and so on. Traditional deposition methods of TIPS-pentacene are drop-casting and spincoating. In this work, drop-casting has proven to lead to better results. A solution of $2 \%$ (in weight) of TIPS-pentacene (Sigma-Aldrich, St. Louis, MS, USA) in a solvent mixture containing 91\% anisole (Sigma-Aldrich) and 9\% decane (Sigma-Aldrich) (in weight) has been used. $50 \mu$ of the semiconductor solution has been deposited by drop-casting, positioning the substrate inside a Petri dish at $3.5^{\circ}$ angle with respect to the horizontal line of a flat hotplate at $50^{\circ} \mathrm{C}$ during 5 minutes. Immediately afterwards, the glass lid has been swapped with filter paper. The substrate has been left to cool down for 1 minute, taking caution that the solvent condensing on the lid does not fall onto the substrate (Figure 2).

HMDS (hexamethyldisilazane) (Sigma-Aldrich) treatment of the substrate surface by vaporization has been used prior to the semiconductor deposition, in order to obtain a nonpolar surface. For that, the substrate has been washed with 2-propanol (IPA) (Sigma-Aldrich), rinsed with deionized water, and air dried. The substrate was treated in a closed Petri dish (sealed with parafilm) together with an $80 \mu \mathrm{L}$ HMDS vial during 4 hours. Figure 3 shows TIPS-pentacene deposition by means of this technique. A dielectric thickness of $300 \mathrm{~nm}$ was achieved.

The electrical characterization of this transistor has been performed with the measuring device described in this work, in order to test its proper operation. A detailed description of this device and the results obtained for this TIP-pentacene OFET are provided in the subsequent sections.
TABLE 1: Substrate specifications (Ossila).

\begin{tabular}{lc}
\hline Size/thickness & $20 \mathrm{~mm} \times 15 \mathrm{~mm} / 725 \pm 25 \mu \mathrm{m}$ \\
\hline Growth/orientation & $\mathrm{CZ} /(100)$ \\
Type/dopant & p-type $/$ Boron \\
Resistivity & $5 \times 10^{-4}$ to $10^{-2} \Omega / \mathrm{cm}$ \\
Front/back surface & Polished/etched \\
Oxide thickness $\left(\mathrm{SiO}_{2}\right)$ & $300 \pm 5 \% \mathrm{~nm}$ \\
Capacitance & $\approx 1.09 \times 10^{-8} \mathrm{~F} / \mathrm{cm}^{2}$ \\
\hline
\end{tabular}

2.2. Architecture of the Measuring Device. Current flowing through a device is traditionally measured with an ammeter and a power supply or a (power or voltage) generator. In recent years, almost all of the top/medium of the range measuring devices have communication ports that allow for control of the instruments (GP-IB, RS232, USB, Ethernet, etc.). In order to plot the I-V characteristics of a device, it is necessary to measure the drain-source current $\left(I_{\mathrm{DS}}\right)$ and the drain-source voltage $\left(V_{\mathrm{DS}}\right)$ for fixed values of the gatesource voltage $\left(V_{\mathrm{GS}}\right)$. That is, a $V_{\mathrm{DS}}$ sweep is performed in a certain interval for every selected value of $V_{\mathrm{GS}}$, while measuring simultaneously $I_{\mathrm{DS}}$ and $V_{\mathrm{DS}}$. This can be performed manually, changing the applied voltage and measuring the current point-by-point. Several programs that allow a generator and a measuring device to be controlled simultaneously using a computer have now come on the market. Nowadays, there are systems that can source a voltage (or electrical current) and read back the associated current (or voltage) simultaneously. These are called source and measurement units (SMUs). A defining SMU characteristic is that they have four-quadrant outputs. They are able to source power in quadrants I and III, as well as sink power in quadrants II and IV. The device developed in this work is based on the NI-PXIe-1073 chassis (National Instruments, Austin, TX, USA). This has been connected to the PCI-e bus of a computer, in order to be controlled (either a laptop or a desktop with Windows 7). This chassis accepts a variety of peripheral module types to be installed. In this work, two NI-PXI-4132 SMU modules (National Instruments) have been selected.

Each one of these SMUs offers up to $\pm 100 \mathrm{~V}$ output (with voltage programming resolution down to $50 \mu \mathrm{V}$, accuracy of $0.025 \%$, and offset of $3 \mathrm{mV}$ ), with current measurement resolution down to $10 \mathrm{pA}$, accuracy of $0.028 \%$, and offset of $1 \mathrm{nA}$. The combination of these features makes this system ideal for the measurement of almost all the characteristics of organic semiconductors. The only inconvenient is that OFET characterization requires current sensitivity into the nanoamperes range and below, which is comparable to the module offset. This demands the use of calibration and compensation techniques to ensure correct measurement.

The SMUs are in turn connected to an EverBeing PE-4 probe station (EverBeing Int'l Corp., Hsinchu, Taiwan), consisting of three test probes, three micropositioners, and three triaxial (coaxial) wires. A test probe is used in each one of the terminals of the transistor (gate, source, and drain). The test probes can be moved in several directions with the micropositioners, which allows for measurement of any type of 


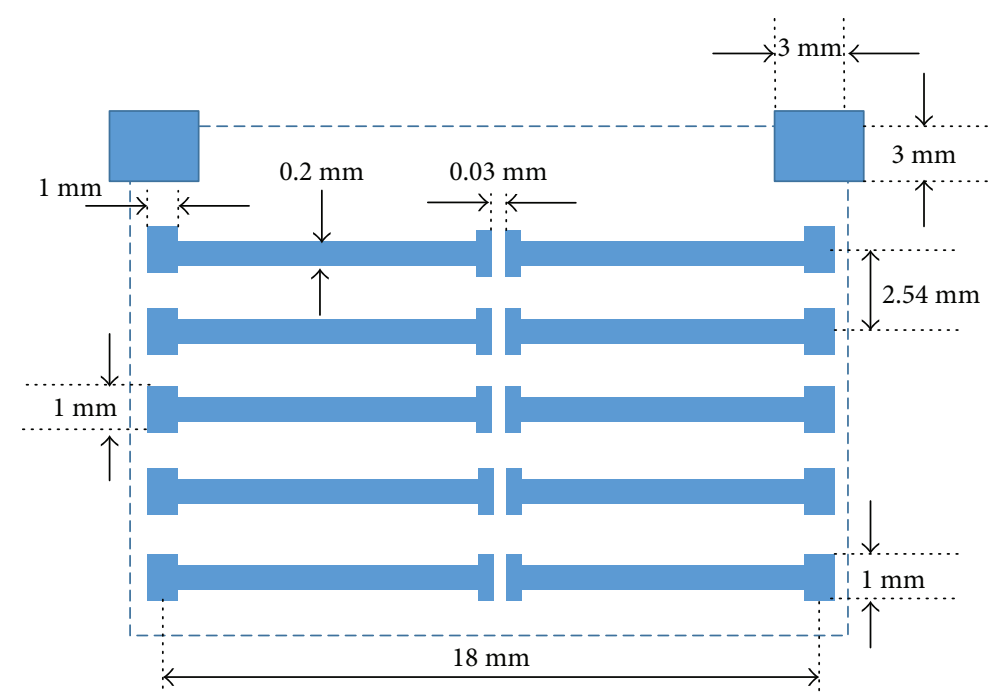

(a)
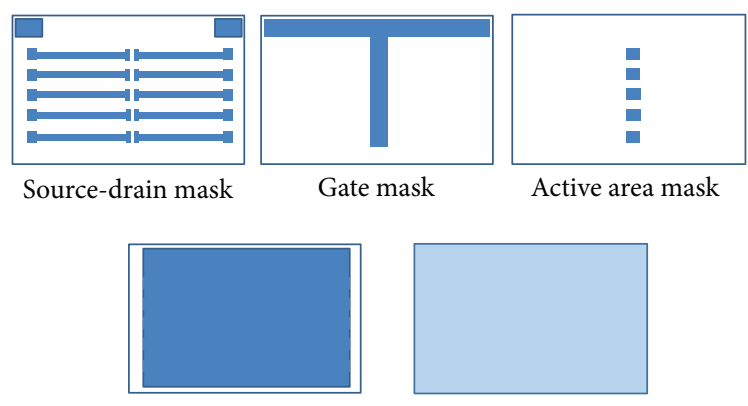

Substrate

(b)

Figure 1: (a) Schematic and dimensions of the substrate. (b) Fabrication layers (source: Ossila).

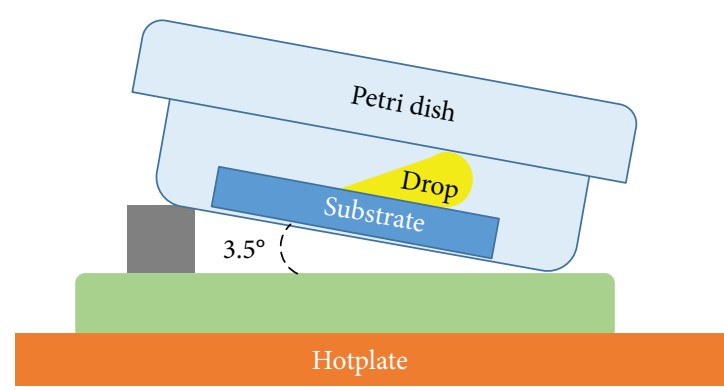

(a)

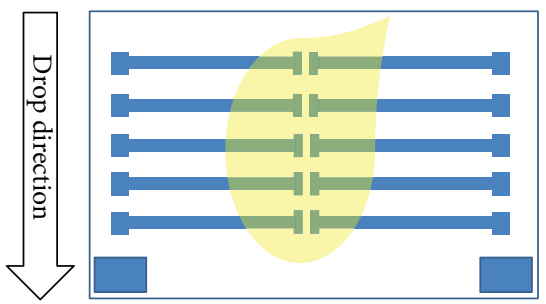

(b)

FIgURE 2: (a) Chip preparation by drop-casting deposition (source: Ossila). (b) Top view of the chip with the pentacene solution drop.

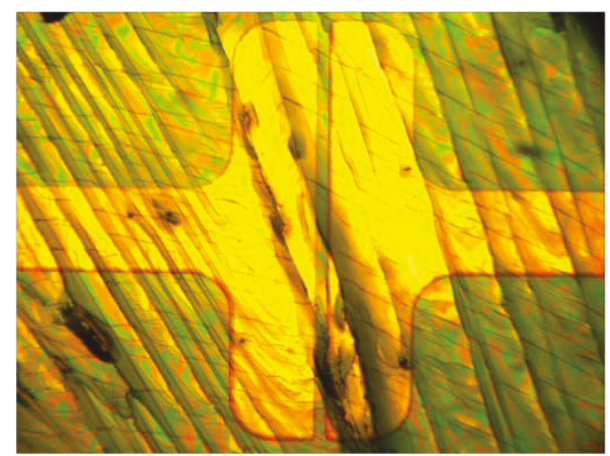

Figure 3: Microscope photograph of one of the OFETs of the Ossila low-density chip after deposition of a TIPS-pentacene layer.

transistor and wafer. The L wires of the SMU-1 and SMU-2 are connected to the source, the $\mathrm{H}$ wire of the SMU-1 is connected to the drain, and the $\mathrm{H}$ wire of the SMU-2 is connected to the gate. Due to the low-level current provided by the organic transistors, 2 wire measurements are performed for the characterization of these transistors. Figure 4 displays the diagram of the complete device. An expansion card has been inserted on the computer motherboard via the
PCI-e bus. The wire that has been used to connect the computer with the PXI chassis is several meters long. This allows the computer to be kept away from the device under test (D.U.T.).

The device has been programmed so that measurements and procedures meet the IEEE 1620-2008 standard for test methods for the characterization of organic transistors and materials [20], and the results can be comparable to those obtained with commercial instruments. The device that has been developed here is able to measure and plot transfer curves ( $I_{\mathrm{DS}}$ versus $V_{\mathrm{DS}}$ ) and to calculate threshold voltage, $V_{\mathrm{T}}$, carrier mobility, $\mu$, (both in the linear regime and the saturation regime), on/off ratio, and so forth. It can also be used to perform measurements in a certain time interval. This can be useful to record the change in a magnitude with time (i.e., $\left.I_{\mathrm{DS}}\right)$ or when a change in the characterization environment of the OFET occurs (i.e., when a pollutant is added).

\section{Results and Discussion}

3.1. Parameter Extraction Program. As previously mentioned, procedures for obtaining the characterization parameters have been developed according to the IEEE 1620-2008 


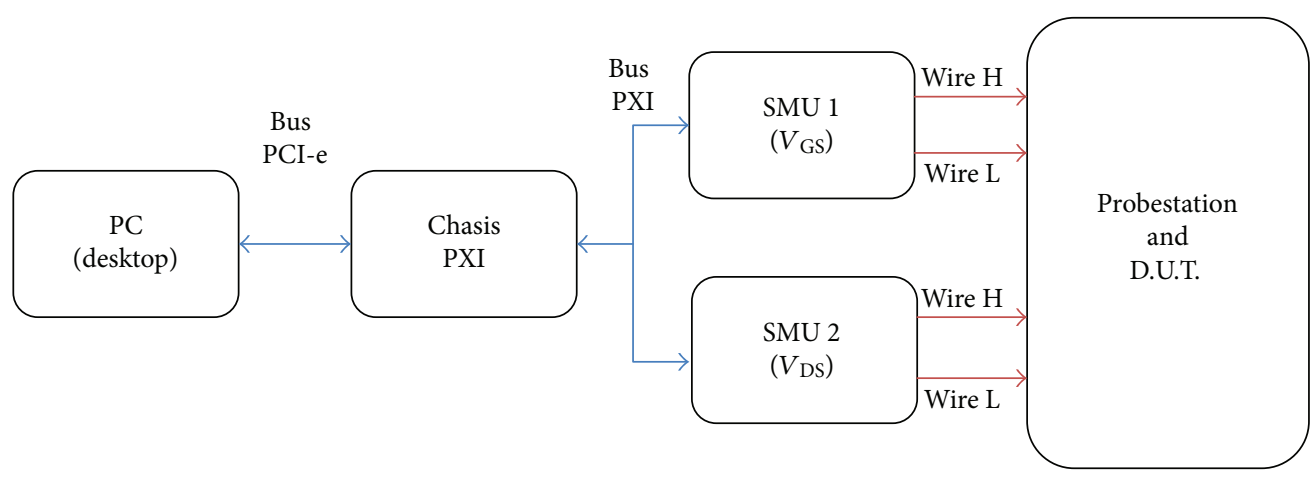

FIgURE 4: Buses and connection diagram.

standard [20]. Test methods are mainly based on the determination of the following parameters:

(i) Transfer curves $\left(\mathrm{I}_{\mathrm{DS}}\right.$ versus $\mathrm{V}_{\mathrm{GS}}$ ), which allow for determination of carrier mobility, $\mu$, and threshold voltage, $\mathrm{V}_{\mathrm{T}}$.

(ii) Output curves ( $I_{\mathrm{DS}}$ versus $\left.V_{\mathrm{DS}}\right)$, which allow for determination of the channel resistance $\left(R_{\mathrm{DS}}\right)$ when the device operates in the ohmic (linear) regime and the characteristics of the saturation regime. These curves can also be used to determine whether the device exhibits FET-like behaviour.

(iii) Gate leakage curves $\left(I_{\mathrm{GS}}\right.$ versus $\left.V_{\mathrm{GS}}\right)$, which allow for characterization of the gate dielectric quality and for determination of the leakage current from the gate to the channel ( $\left.I_{\text {leakage }}\right)$.

The test system is controlled by a program that has been developed using LabVIEW (National Instruments). Four modules of this system are currently available: $I_{\mathrm{DS}}$ versus $V_{\mathrm{DS}}$ measurement, $I_{\mathrm{DS}}$ versus $V_{\mathrm{GS}}$ measurement, $I_{\mathrm{DS}}$ versus time measurement, and $I_{\mathrm{GS}}$ versus $V_{\mathrm{GS}}$ measurement. Figure 5 shows the flowchart of the different measurement procedures of the test system.

3.1.1. $I_{D S}$ versus $V_{D S}$ Module. This module is based on the measurement of the drain characteristics. The drain current $\left(I_{\mathrm{DS}}\right)$ is measured versus the drain voltage $\left(V_{\mathrm{DS}}\right)$ for different gate voltages $\left(V_{\mathrm{GS}}\right)$. Figure 6 shows a screenshot of this module and Figure 7 displays the output curves corresponding to the TIPS-pentacene OFET described previously. Configuration of the following parameters is required to run this module: $V_{\mathrm{DS}}$ start, $V_{\mathrm{DS}}$ stop, $V_{\mathrm{DS}}$ step, $I_{\mathrm{DS}}$ current limit, and $V_{\mathrm{DS}}$ delay (dwell time). The dwell time corresponds to the duration from the time when the measurement voltage is applied to the time when the measurement is recorded. This parameter is used to minimize measurement errors due to current transients.

Two types of tests can be performed. The first test ( $V_{\mathrm{GS}}$ sweep) consists of a scan between two selected values of $V_{\mathrm{GS}}$, that is, the $V_{\mathrm{DS}}$ value is swept between two limits for each $V_{\mathrm{GS}}$ step. As a result, a set of curves is obtained. In the second test ( $V_{\mathrm{GS}}$ fixed), a scan is performed for a fixed $V_{\mathrm{GS}}$. As a result, a single characteristic curve is obtained.
3.1.2. $I_{D S}$ versus $V_{G S}$ Module. This module is based on a transfer measurement $\left(I_{\mathrm{DS}}\right.$ versus $V_{\mathrm{GS}}$ for a fixed $\left.V_{\mathrm{DS}}\right)$. This curve allows for the determination of additional parameters like threshold voltage $\left(V_{\mathrm{T}}\right)$, on/off ratio $\left(I_{\mathrm{on}} / I_{\text {off }}\right)$, subthreshold swing $(S)$ and carrier mobility $(\mu)$. Moreover, measurements can be performed either in the linear regime or in the saturation regime.

(1) Linear Regime. Two types of scans can be performed for the determination of $I_{\mathrm{DS}}$ versus $V_{\mathrm{GS}}$ in the linear regime: simple (single linear scan) and hysteresis (there-and-back scan). The threshold voltage $\left(V_{\mathrm{T}}\right)$ corresponds to the interception with the $x$-axis ( $V_{\mathrm{GS}}$ ) of the straight line with the same slope in the linear part of the $I_{\mathrm{DS}}-V_{\mathrm{GS}}$ curve (Figure 8(a)). The on/ off ratio $\left(I_{\text {on }} / I_{\text {off }}\right)$ can also be determined by dividing the maximum $I_{\mathrm{DS}}$ value by the minimum $I_{\mathrm{DS}}$ value obtained from the transfer curve.

The carrier mobility $(\mu)$ of the semiconductor can also be derived through transfer curve measurement of the device, in combination with geometric and electrical parameters of the OFET that are additional inputs to be provided by the user. Carrier mobility also requires knowledge of the slope of the linear part of the transfer curve. The program developed here allows for the determination of carrier mobility by means of three different methods:

(i) Backward derivative (B.D.):

$$
\mu(\text { B.D. })=\frac{1}{\mathrm{Cox}} \cdot \frac{1}{V_{\mathrm{DS}}} \cdot \frac{L}{W} \cdot \frac{I_{\mathrm{DS}}(V+h)-I_{\mathrm{DS}}(V)}{h},
$$

(ii) Two-point derivative (T.P.):

$$
\mu(\mathrm{T} . \mathrm{P} .)=\frac{1}{\mathrm{Cox}} \cdot \frac{1}{V_{\mathrm{DS}}} \cdot \frac{L}{W} \cdot \frac{I_{\mathrm{DS}}(V+h)-I_{\mathrm{DS}}(V-h)}{2 h},
$$

(iii) Least square fit derivative (alpha):

$$
\mu(\alpha)=\frac{1}{\operatorname{Cox}} \cdot \frac{1}{V_{\mathrm{DS}}} \cdot \frac{L}{W} \cdot \alpha\left(V_{\mathrm{GS}}\right),
$$

where Cox is the capacitance per unit area, $V_{\mathrm{DS}}$ is the voltage measured between the drain electrode and the source 


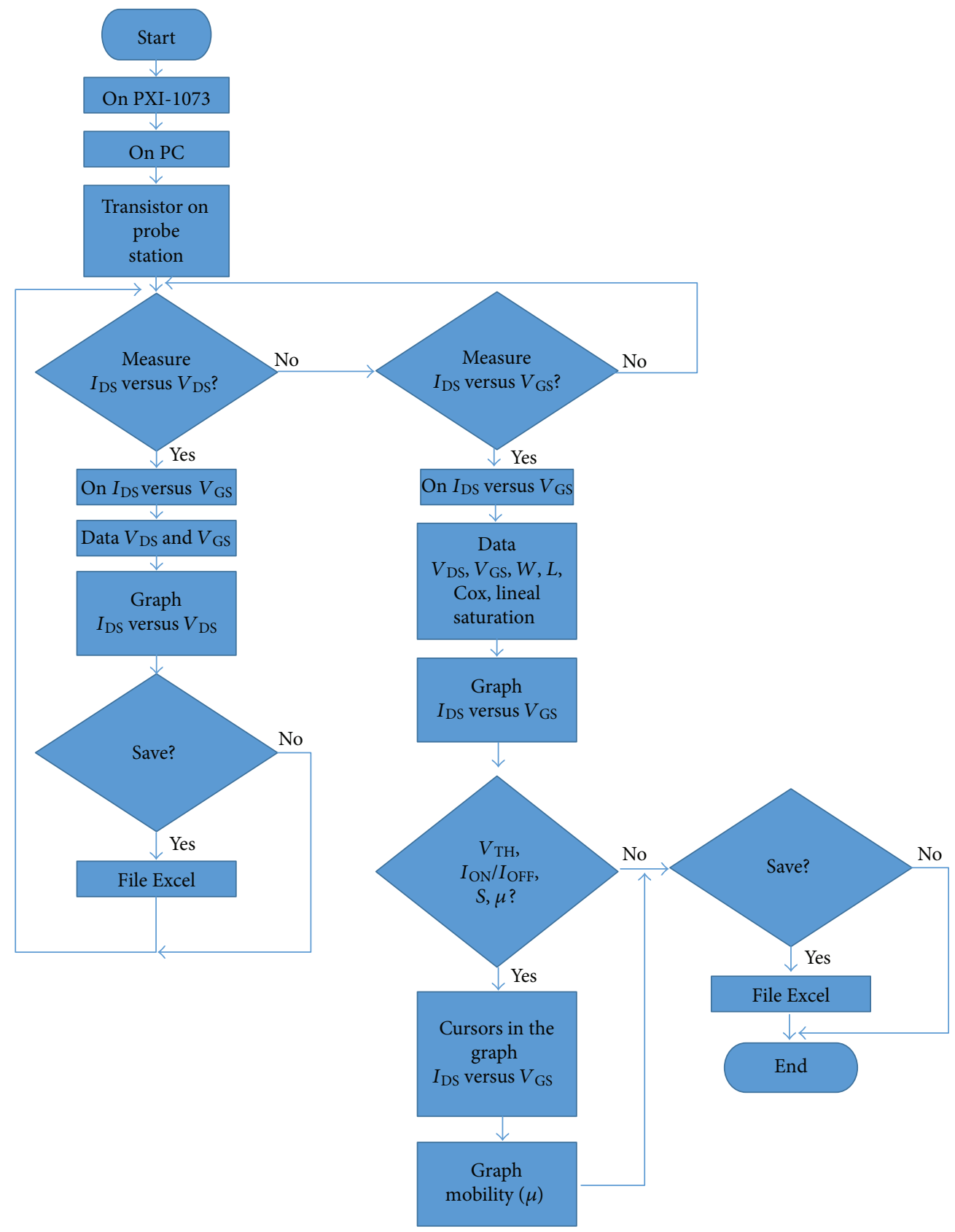

Figure 5: Flowchart of the test system.

electrode (fixed value), $L$ is the channel length, $W$ is the channel width, $I_{\mathrm{DS}}$ is the current flow measured through the drain electrode, and $h$ is the step between two consecutive $V_{\mathrm{Gs}}$. The slope $\alpha$ is determined with three points of the curve by the leas-squares method. Figure 9(a) shows an example of the determination of carrier mobility using the backward derivative method.

(2) Saturation Regime. The OFET behaviour in the saturation regime can be properly displayed in an I-V diagram, where the square root of $I_{\mathrm{DS}}$ and the logarithm of $I_{\mathrm{DS}}$ are plotted versus $V_{\mathrm{GS}}$. Figure $8(\mathrm{~b})$ shows an example of this diagram. The threshold voltage $\left(V_{\mathrm{T}}\right)$ can be obtained similarly to that of the linear regime, using the curve of the square root of the drain current $\left(I_{\mathrm{DS}}\right)$ versus the gate-source voltage $\left(V_{\mathrm{GS}}\right)$. The on/off ratio $\left(I_{\text {on }} / I_{\text {off }}\right)$ can be obtained from the maximum and minimum values of the curve of the logarithm of $I_{\mathrm{DS}}$ versus $V_{\mathrm{GS}}$. The subthreshold swing $(S)$ corresponds to the maximum slope of the $\log \left(I_{\mathrm{DS}}\right)$ versus $V_{\mathrm{GS}}$ curve in the saturation regime. Carrier mobility $(\mu)$ in the saturation regime can also be obtained similarly to that of the linear regime (Figure 9(b)).

3.1.3. Measurements as a Function of Time. OFETs in general have been demonstrated as effective sensors in both liquid $\left(\mathrm{H}^{+}\right.$, glucose, hydrogen peroxide, $\mathrm{Cu}(\mathrm{II})$ ions, etc.) and gaseous environments (water vapor, chloroform, ammonia, 


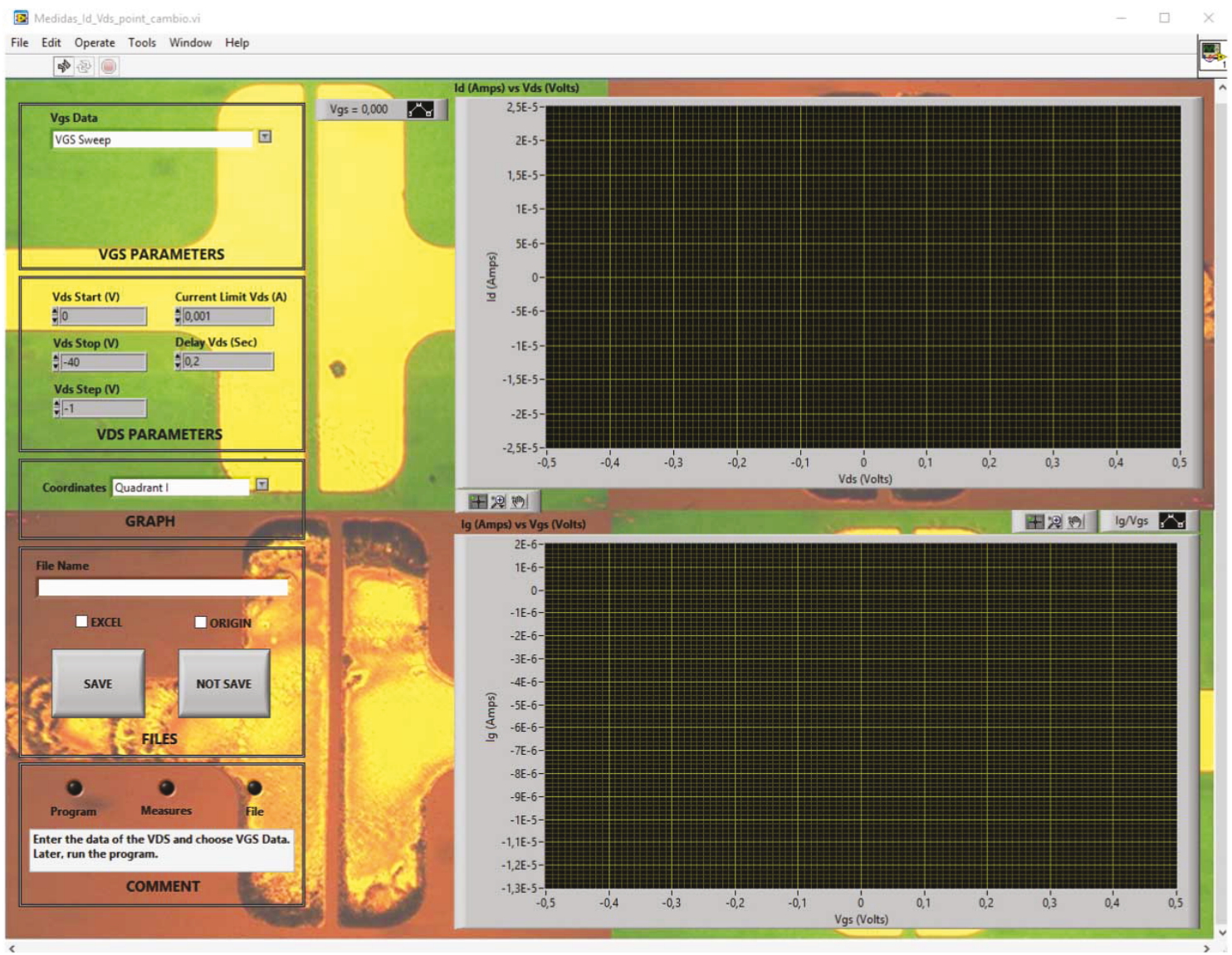

FIGURE 6: Screenshot of the program running the $I_{\mathrm{DS}}$ versus $V_{\mathrm{DS}}$ module.

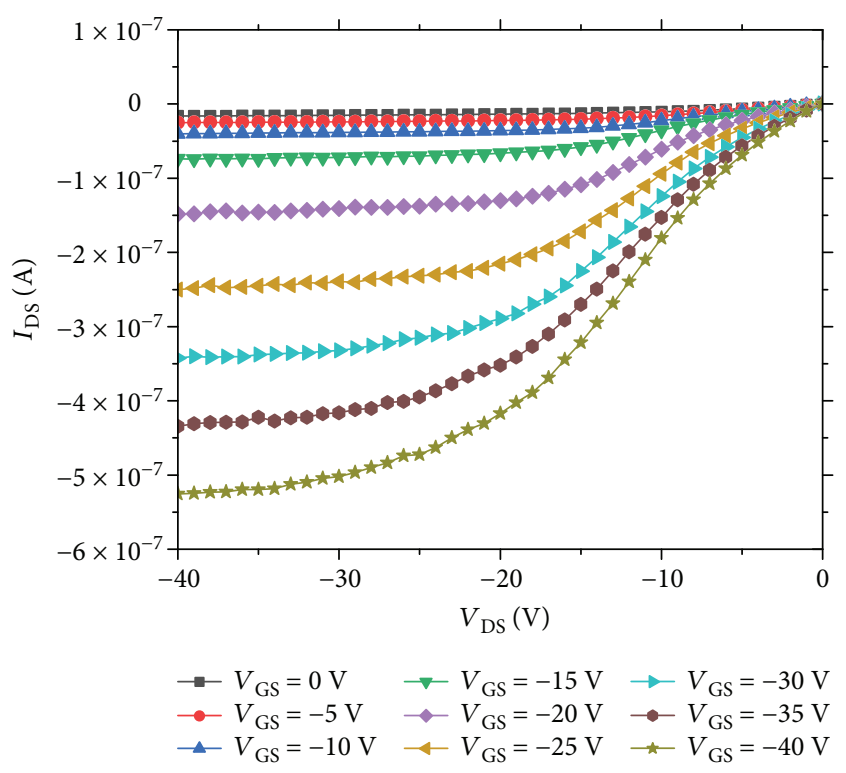

Figure 7: $I_{\mathrm{DS}}-V_{\mathrm{DS}}$ curves of the TIPs-pentacene OFET.

etc.) [15]. Response of these sensors is usually not immediate, since the semiconductor has to react with the analyte. In order to study the OFET behaviour in transients, a module that monitors changes of $I_{\mathrm{DS}}$ with time has been included in the characterization program. By providing values of $V_{\mathrm{GS}}, V_{\mathrm{DS}}$, and storage time, $I_{\mathrm{DS}}$ values are displayed in real time. Furthermore, since sensing experiments are usually performed while changing the analyte concentration, the program developed here can also indicate when the change in concentration occurs.

3.1.4. I $I_{G S}$ versus $V_{G S}$ Measurements. This is the most demanding measurement of the acquisition system, since $I_{\mathrm{GS}}$ is very small and several orders of magnitude below $I_{\mathrm{DS}}$ (which is also small by itself). Thus, to perform this measurement, the sensitivity in the determination of $V_{\mathrm{GS}}$ by the SMU must be enhanced as much as possible. This in turn leads to a significant increase in the measurement time. Figure 10 shows an example of the gate leakage measurement performed with this device.

3.2. Characterization of the TIPS-Pentacene OFET. The main characteristic parameters of the TIPS-pentacene OFET that have been determined in this work according to the IEEE 1620-2008 standard are summarized in Table 2. The threshold voltage that has been obtained is in good agreement with that reported by Ossila for this transistor. A better carrier mobility has been found than that reported by Ossila [21].

3.3. Comparison with a Standard Semiconductor Test System. A comparative measurement has been made between the 




(a)

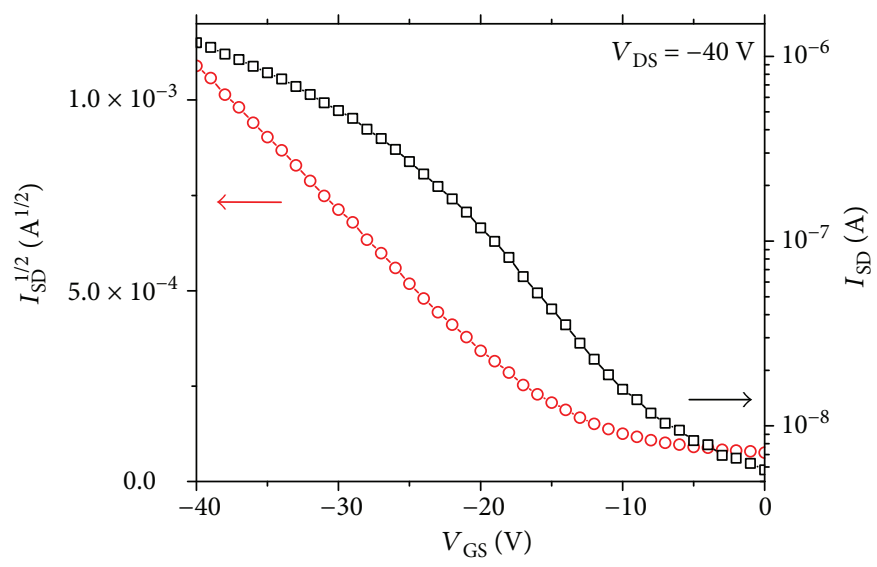

(b)

FIGURE 8: $I_{\mathrm{DS}}-V_{\mathrm{GS}}$ curves of the TIPs-pentacene OFET in (a) the linear regime and (b) the saturation regime.



(a)

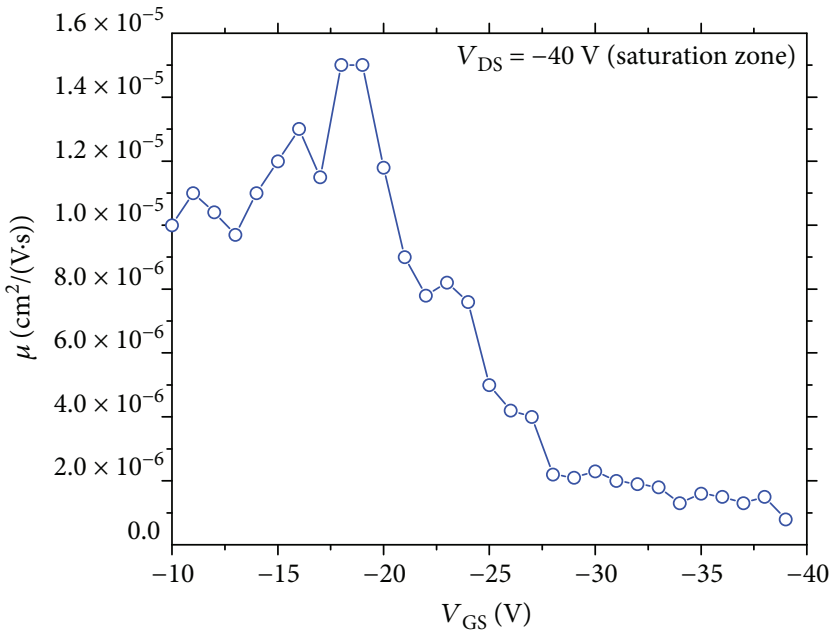

(b)

FIgURE 9: Carrier mobility values of the TIPs-pentacene OFET determined with the backward derivative method in (a) the linear regime and (b) the saturation regime.

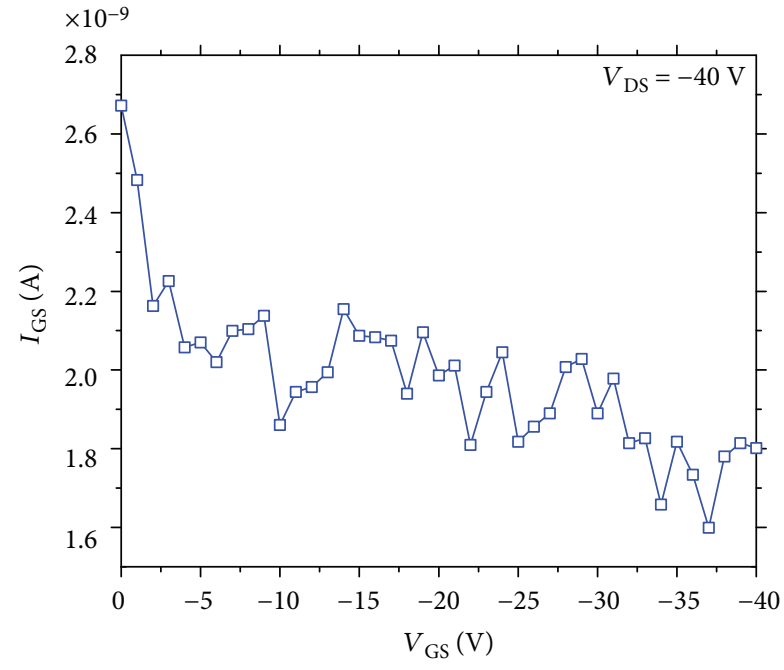

FIGURE 10: $I_{\mathrm{GS}}-V_{\mathrm{GS}}$ values for a fixed $V_{\mathrm{DS}}$ of the TIPs-pentacene OFET. equipment described in this work and a high-quality semiconductor characterization equipment (Keithley 4200A-SCS Parameter Analyzer) to check its reliability. Figure 11 shows the $I_{\mathrm{GS}}$ versus $V_{\mathrm{GS}}$ curves obtained with these two types of equipment. An error of $4 \%$ has been found in the results of our measuring module compared to those of the Keithley equipment.

\section{Conclusions}

The unique properties of organic semiconductors cause measurement issues that are not typically encountered with inorganic semiconductors. This renders difficult the electrical characterization of organic transistors, since commercial devices have been all designed for characterizing Si-based transistors. It is therefore of great interest to develop a device that can be used for specifically testing organic transistors. In this work, a low-cost device which allows for systematic characterization of OFETs has been developed. This measuring device allows for easy determination of the minimum 
TABLE 2: Characterization of the TIPS-pentacene OFET according to the IEEE1620-2008 standard.

\begin{tabular}{|c|c|c|c|}
\hline Parameter & Symbol & Value & Ossila \\
\hline Stray capacitance & $C_{\mathrm{GD}}$ & $697.38 \mathrm{pF}$ at $100 \mathrm{KHz}$ & - \\
\hline Stray capacitance & $C_{\mathrm{GS}}$ & $685.28 \mathrm{pF}$ at $100 \mathrm{KHz}$ & - \\
\hline Conductance & $g_{\mathrm{s}}=\partial \frac{I_{\mathrm{DS}}}{\partial} V_{\mathrm{DS}}$ & $\begin{array}{l}\text { Saturation region }=2 \mathrm{pA} / \mathrm{V} \\
\text { Linear region }=28 \mathrm{pA} / \mathrm{V}\end{array}$ & - \\
\hline Dwell time & & $500 \mathrm{~ms}$ & - \\
\hline Environmental conditions & & $26^{\circ} \mathrm{C}, 60 \% \mathrm{RH}$, dark, air & - \\
\hline Carrier mobility & $\mu$ & $\begin{array}{l}\text { Saturation region }=15.10^{-6} \mathrm{~cm}^{2} / \mathrm{V} \cdot \mathrm{s} \\
\text { Linear region }=135 \cdot 10^{-6} \mathrm{~cm}^{2} / \mathrm{V} \cdot \mathrm{s}\end{array}$ & $\begin{array}{c}0.38 \times 10^{-6} \\
-\end{array}$ \\
\hline Gate leakage current & $I_{\mathrm{GS}}$ & $125 \mathrm{pA}$ & - \\
\hline On/off ratio & & 32.91 & - \\
\hline Subthreshold swing & & $0.335 \log (\mathrm{A}) / \mathrm{V}\left(V_{\mathrm{DS}}=-40 \mathrm{~V}, V_{\mathrm{GS}}=-5 \mathrm{~V}\right)$ & - \\
\hline Threshold voltage & $V_{\mathrm{T}}$ & $-11.79 \mathrm{~V}\left(V_{\mathrm{DS}}=-25 \mathrm{~V}\right)$ & -12.99 \\
\hline Transconductance & $g_{\mathrm{m}}=\partial \frac{I_{\mathrm{DS}}}{\partial} V_{\mathrm{GS}}$ & $17.25 \mathrm{pA} / \mathrm{V}\left(V_{\mathrm{DS}}=-25 \mathrm{~V}\right)$ & - \\
\hline
\end{tabular}



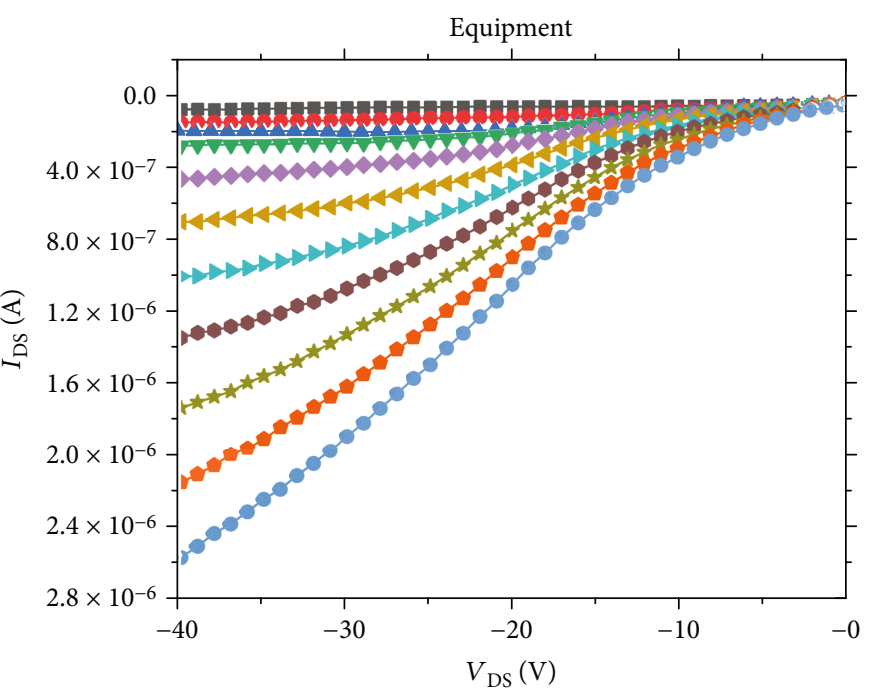

FIGURE 11: $I_{\mathrm{DS}}-V_{\mathrm{DS}}$ curves obtained with the Keithley 4200 A-SCS equipment and the equipment described in this work.

reporting parameters as specified in the IEEE 1620-2008 standard. As an example, this device has been used for the characterization of TIPS-pentacene OFETs.

\section{Conflicts of Interest}

The authors declare that they have no conflicts of interest.

\section{Authors' Contributions}

Eduardo García-Breijo conceived and designed the measuring module. José Vicente Lidón-Roger constructed the measuring module. Laura Contat-Rodrigo and Eduardo
García-Breijo designed the experiments for the electrical characterization of the transistors and for DCP sensing. Clara Pérez-Fuster and José Vicente Lidón-Roger fabricated the transistors and performed the electrical measurements. Clara Pérez-Fuster performed the experimental tests for DCP sensing. All the authors analyzed the data and wrote the paper.

\section{Acknowledgments}

All financial support from the Spanish Government and FEDER funds (MAT2015-64139-C4-3-R (MINECO/ FEDER)) and the Generalitat Valenciana (GVA funds) (AICO/2015/103) is gratefully acknowledged. 


\section{References}

[1] B. Kumar, B. K. Kaushik, and Y. S. Negi, "Organic thin film transistors: structures, models, materials, fabrication, and applications: a review," Polymer Reviews, vol. 54, no. 1, pp. 33-111, 2014.

[2] Ö. Abanoz and C. Dimitrakopoulos, "Recent advances in organic field effect transistors," Turkish Journal of Physics, vol. 38, pp. 497-508, 2014.

[3] Z. R. Li, Organic Light-Emitting Materials and Devices, CRC press Taylor \& Francis Group, Boca Raton, 2015.

[4] C. Murawski, K. Leo, and M. C. Gather, "Efficiency roll-off in organic light-emitting diodes," Advanced Materials, vol. 25, no. 47, pp. 6801-6827, 2013.

[5] N. Kaur, M. Singh, D. Pathak, T. Wagner, and J. M. Nunzi, "Organic materials for photovoltaic applications: review and mechanism," Synthetic Metals, vol. 190, pp. 20-26, 2014.

[6] J. Yu, Y. Zheng, and J. Huang, "Towards high performance organic photovoltaic cells: a review of recent development in organic photovoltaics," Polymer, vol. 6, no. 9, pp. 2473-2509, 2014.

[7] S. M. Lee, J. H. Kwon, S. Kwon, and K. C. Choi, "A review of flexible OLEDs toward highly durable unusual displays," IEEE Transactions on Electron Devices, vol. 64, no. 5, pp. 1922-1931, 2017.

[8] L. Torsi, M. Magliulo, K. Manoli, and G. Palazzo, "Organic field-effect transistor sensors: a tutorial review," Chemical Society Reviews, vol. 42, no. 22, pp. 8612-8628, 2013.

[9] P. Lin and F. Yan, "Organic thin-film transistors for chemical and biological sensing," Advanced Materials, vol. 24, no. 1, pp. 34-51, 2012.

[10] K. J. Baeg, M. Caironi, and Y. Y. Noh, "Toward printed integrated circuits based on unipolar or ambipolar polymer semiconductors," Advanced Materials, vol. 25, no. 31, pp. 4210-4244, 2013.

[11] J. S. Chang, A. F. Facchetti, and R. Reuss, "Guest editorial organic/printed electronics: a circuits and systems perspective," IEEE Journal on Emerging and Selected Topics in Circuits and Systems, vol. 7, no. 1, pp. 1-6, 2017.

[12] S. Khan, L. Lorenzelli, and R. S. Dahiya, "Technologies for printing sensors and electronics over large flexible substrates: a review," IEEE Sensors Journal, vol. 15, no. 6, pp. 31643185, 2015.

[13] S. H. Kim, K. Hong, W. Xie et al., "Electrolyte-gated transistors for organic and printed electronics," Advanced Materials, vol. 25, no. 13, pp. 1822-1846, 2013.

[14] I. Kymissis, Organic Field-Effect Transistors. Theory, Fabrication and Characterization, Springer, New York, USA, 2009.

[15] J. T. Mabeck and G. G. Malliaras, "Chemical and biological sensors based on organic thin-film transistors," Analytical and Bioanalytical Chemistry, vol. 384, no. 2, pp. 343-353, 2006.

[16] I. Manunza, Organic Field-Effect Devices for Detecting Chemical and Physical Parameters, Department of Electrical and Electronic Engineering. University of Cagliari, Italy, 2007.

[17] P. Lienerth, S. Fall, P. Lévêque, U. Soysal, and T. Heiser, "Improving the selectivity to polar vapors of OFET-based sensors by using the transfer characteristics hysteresis response," Sensors and Actuators B: Chemical, vol. 225, pp. 90-95, 2016.

[18] A. N. Sokolov, M. E. Roberts, O. B. Johnson, Y. Cao, and Z. Bao, "Induced sensitivity and selectivity in thin-film transistor sensors via calixarene layers," Advanced Materials, vol. 22, no. 21, pp. 2349-2353, 2010.

[19] S. Ochiai, K. Palanisamy, S. Kannappan, and P. K. Shin, "Pentacene active channel layers prepared by spin-coating and vacuum evaporation using soluble precursors for OFET applications," ISRN Condensed Matter Physics, vol. 2012, Article ID 313285, 7 pages, 2012.

[20] Working group for electronic and molecular electronics, "IEEE Std 1620-2008-IEEE Standard for test methods for the characterization of organic transistors and materials.

[21] Ossila. https://cdn.shopify.com/s/files/1/0823/0287/files/pre fab_specv2.pdf. 


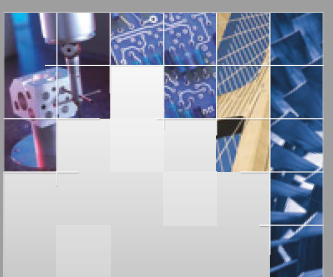

\section{Enfincering}
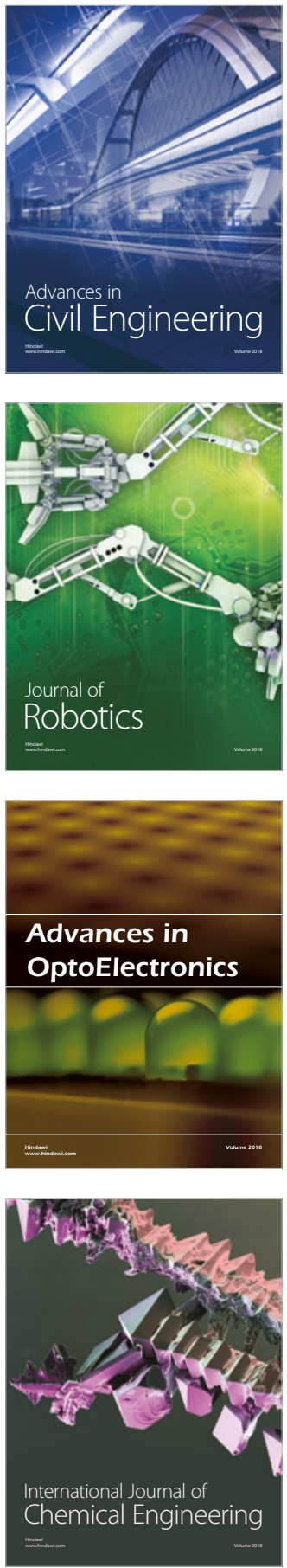

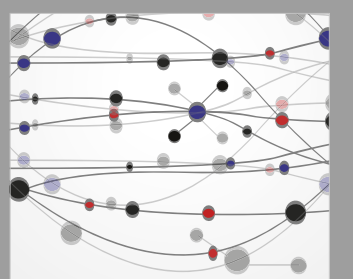

\section{Rotating \\ Machinery}

The Scientific World Journal

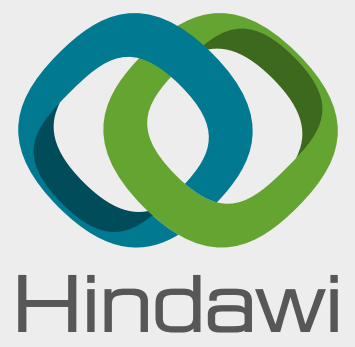

Submit your manuscripts at

www.hindawi.com

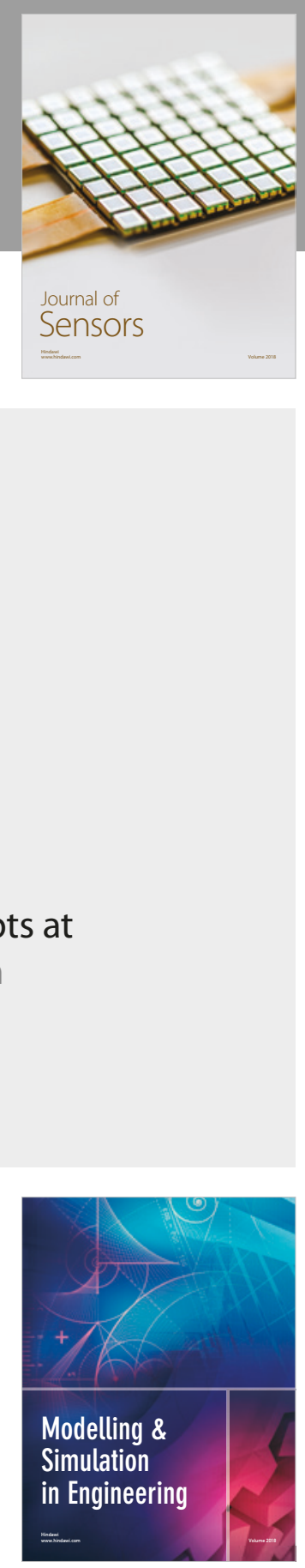

\section{Advances \\ Multimedia}
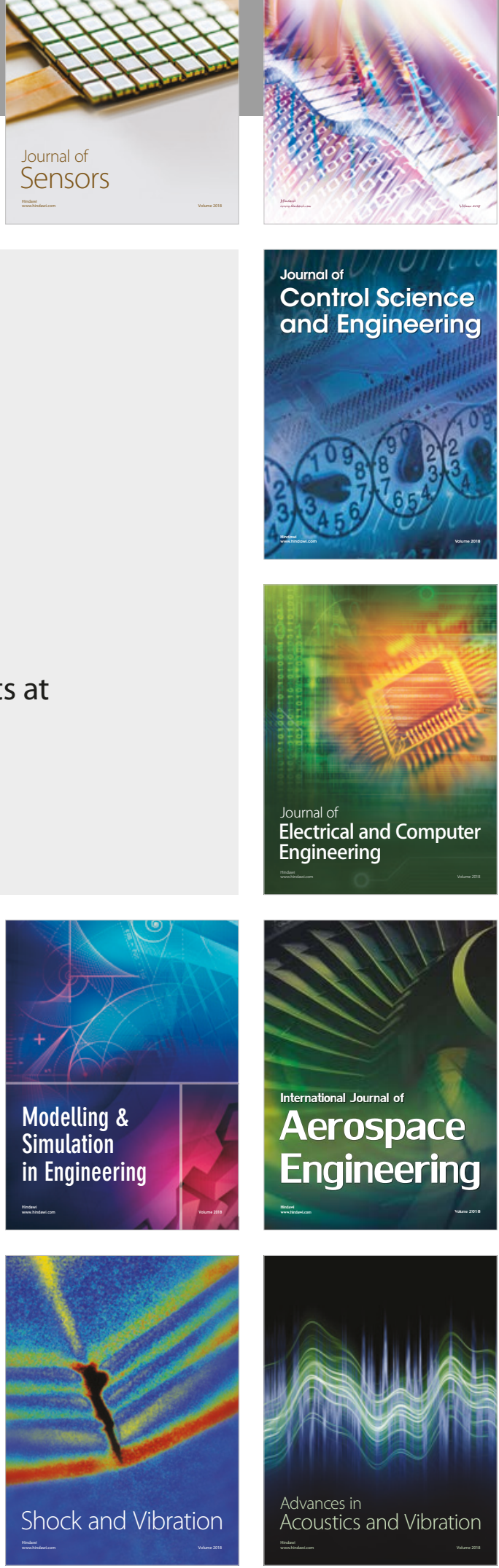